\title{
A VCO WITH HARMONIC SUPPRESSED AND OUTPUT POWER IMPROVED USING DEFECTED GROUND STRUCTURE
}

\author{
HAIWEN LIU a,b,, XIAOWEI SUN ${ }^{b}$ and ZHENGFAN LI ${ }^{a}$ \\ aD epartment of Electronic Engineering, Shanghai Jiaotong U niversity, Shanghai 200030, China; \\ ${ }^{b}$ Shanghai Institute of Microsystem and Information Technology, Chinese Academy of Sciences, \\ Shanghai 200050, China
}

(Received 14 January 2003; In final form 21 March 2003)

\begin{abstract}
In this paper, a novel defected ground structure (DGS) is proposed for suppressing harmonic and increasing the output power of a voltage-controlled oscillator (VCO) in microwave circuits. The DGS is formed with connecting in parallel two periodic structures which have different center frequencies (in a ratio of 2:3) of the stopband and provides the bandgap characteristic in certain frequency bands. Simulated and experimental results show that the microstrip line with DGS has a wide low-pass band for the fundamental frequency and a stopband for the second harmonic with good performance. To evaluate the effects of DGS on microwave VCOs, two GaAs field-effect transistor (FET) V COs have been designed and fabricated. One of them has a $50 \Omega$ microstrip line with DGS at the output section, while the other has only a $50 \Omega$ straight line. M easured results show that DGS suppresses the second harmonic more than $-20 \mathrm{dBm}$ at the output and yields improved output power by $3-5 \%$.
\end{abstract}

Keywords: Voltage-controlled oscillator; Harmonic suppressed; Defected ground structure

\section{INTRODUCTION}

Recently, there has been an increasing interest in studying the microstrip line with various periodic structures including photonic bandgap (PBG) [1-4] and defected ground structure (DGS) [5-9] in the design of microwave circuits such as filters, divider, amplifiers and so on. DGS, which is realized by etching only a row of defects on the ground plane, is also a kind of periodic structure. DGSs are much more easily designed and fabricated and occupy less area than PBGs do. A nd DGSs have similar and/or superior characteristics to the conventional PBGs. M oreover, DGSs have prominent advantages in extending their applicability to other microwave circuits.

One important parameter of designing microwave VCO is output power. The output power mainly depends on the active device, bias conditions, matching networks, and so on. One of the methods to improve the output power is to terminate the harmonics at the output. A mong the harmonics, the second harmonic is especially in want of controlling,

\footnotetext{
* Corresponding author. Tel.: (86-21) 629 33001; E-mail: haiwen_liu@sjtu.edu.cn/haiwen_liu@ hotmail.com
} 
because its magnitude is relatively larger than the other harmonics. The controlling of the second harmonic is usually done by adding a short-circuited stub which is approximately one-quarter wavelength long at the fundamental frequency. A Iternatively, chip capacitors with a self-resonance near the second harmonic can be used [7].

In this paper, a novel DGS is proposed and formed with connecting in parallel two periodic structures which have different center frequencies (in a ratio of 2:3). The frequency characteristics of the DGS are discussed by three-dimensional field analysis. It is shown that a microstrip line with the DGS results in a wide low-pass band for the fundamental frequency and a stopband for the second harmonic with good performance. Therefore, a new technique is provided to improve the output power and suppress harmonics in VCOs using the proposed DGS. To confirm the validity of the technique, two GaAs FET VCOs have been fabricated and measured. One has a $50 \Omega$ microstrip line with DGS at the output section, while the other has a $50 \Omega$ straight line only. M easured results show that DGS rejects the second harmonic at the output and yields improved output power by 3-5\%.

\section{DGS DESIGN AND CHARACTERISTICS}

A hn [5] has reported that DGS etched in backside metallic ground plane disturbs the shield current distribution in the ground plane. This disturbance increases the effective capacitance and inductance of a transmission line, respectively, so that DG S can provide cutoff frequency in certain frequency without any periodic arrays. M oreover, the microstrip line with DGS provides a bandgap effect. Figure 1 shows one etched lattice shape of three proposed DGSs, which is located on the backside metallic ground plane and placed under the $50 \Omega$ microstrip line. The unit DGS pattern is composed of two different PBG structures (which have the center frequencies of 20 and $30 \mathrm{GHz}$ in the rejection band; we call it a dual-periodic $P B G$ ) in parallel on the ground plane. The substrate used has a dielectric constant of 9.8 with a thickness of $0.3 \mathrm{~mm}$. A ccording to the method in R ef. [1] to design the lattices of PB $\mathrm{ss}$, the periodic distances are determined by $p=\lambda_{\mathrm{g}} / 2$, where $\lambda_{\mathrm{g}}$ is the guided wavelength of the standards microstrip line. The length of the rectangle lattice is optimized by $a=p / 2$. Therefore, when the stopband center frequency of PBG is selected to be $30 \mathrm{GHz}$, then $p_{1}=1.88 \mathrm{~mm}$ and $a_{1}=0.95 \mathrm{~mm}$. When the stopband center frequency of PBG is selected to be $20 \mathrm{GHz}$, then

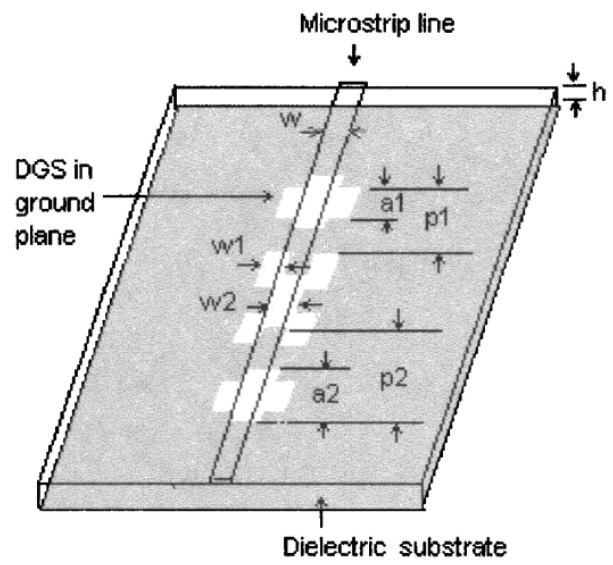

FIGURE 1 Three-dimensional view of one of the proposed DGSs. 
$p_{2}=2.86 \mathrm{~mm}$ and $a_{2}=1.43 \mathrm{~mm}$. The width of the rectangle lattices $w_{1}$ and $w_{2}$ are less than $a_{1}$ and $a_{2}$, respectively. The conductor strip on the top plane has a width $w=0.3 \mathrm{~mm}$, corresponding to a $50 \Omega$ line for the conventional microstrip.

The patterns of the three proposed DGSs are shown in Figure 2. The dimensions of the DGS can be obtained from the above analysis. The patter a has five $0.95 \mathrm{~mm} \times 0.75 \mathrm{~mm}$ $\left(a_{1} \times w_{1}\right)$ rectangles of $1.88 \mathrm{~mm}$ period $\left(p_{1}\right)$ and three $1.5 \mathrm{~mm} \times 0.6 \mathrm{~mm}\left(a_{2} \times w_{2}\right)$ rectangles of $2.88 \mathrm{~mm}$ period $\left(p_{2}\right)$ in parallel. The patter $b$ has five $1.1 \mathrm{~mm} \times 0.6 \mathrm{~mm}$ rectangles of $1.88 \mathrm{~mm}$ period and three $1.4 \mathrm{~mm} \times 0.5 \mathrm{~mm}$ rectangles of $2.82 \mathrm{~mm}$ period in parallel. The patter $c$ has seven $1.1 \mathrm{~mm} \times 0.6 \mathrm{~mm}$ rectangles of $1.88 \mathrm{~mm}$ period and five $1.4 \mathrm{~mm} \times$ $0.5 \mathrm{~mm}$ rectangles of $2.82 \mathrm{~mm}$ period in parallel.

The frequency characteristics of microstrip lines with the proposed DGS are simulated by three-dimensional field analysis software Sonnet Lite 8.0 and are shown in Figure 3. The simulated results show wide low-pass filter characteristics with good performances, as expected. Existing of the cutoff frequency means that employing the DGS section increases the effective permittivity so that the effective inductance of a microstrip line is also increased. From the simulated results shown in Figure 3, the output power of the VCO is not degraded by DGS because of excellent $S_{11}$ and $S_{22}$ at the operating frequency band which is from 12.2 to $12.5 \mathrm{GHz}$. It should be noted that the stopband begins abruptly from about $19.5 \mathrm{GHz}$ and the frequency band of the second harmonic to be tuned is from 24.4 to $25 \mathrm{GHz}$ in VCO. In Figure 3, it is shown that the microstrip lines with the proposed DGS provide the wide low-pass band from 1 to $12 \mathrm{GHz}$ for the fundamental frequency with $\mathrm{S}_{21}$ less than $-0.5 \mathrm{~dB}$. However, their stopband characteristics for the second harmonic are obviously different. A mong the three DGSs, the DGS made up of patter $c$ provides the best stopband characteristic for the second harmonic with $S_{21}$ more than $-40 \mathrm{~dB}$ from 21.5 to $26.5 \mathrm{GHz}$ while the DGS made up of patter $\mathrm{b}$ is with $\mathrm{S}_{21}$ more than $-40 \mathrm{~dB}$ from 21.5 to $26 \mathrm{GHz}$ and the DGS made up of patter $a$ is with $S_{21}$ more than $-40 \mathrm{~dB}$ from 21 to $24 \mathrm{GHz}$. On the contrary, the DGS made up of patter c occupies the largest area. Frequency characteristics and occupied area taken into account, the DGS made up of patter $\mathrm{b}$ is selected and used to suppress the second harmonic and increase the output power in $\mathrm{VCO}$.

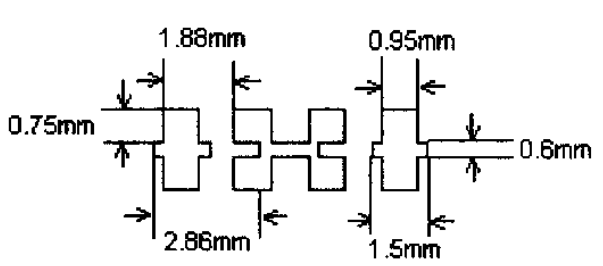

a

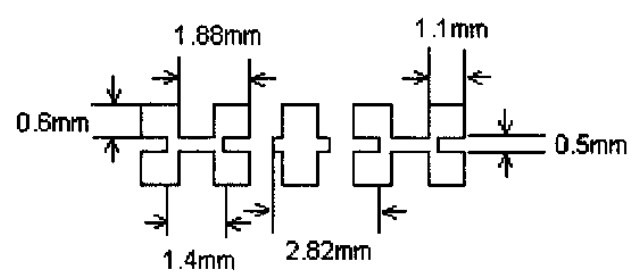

$b$

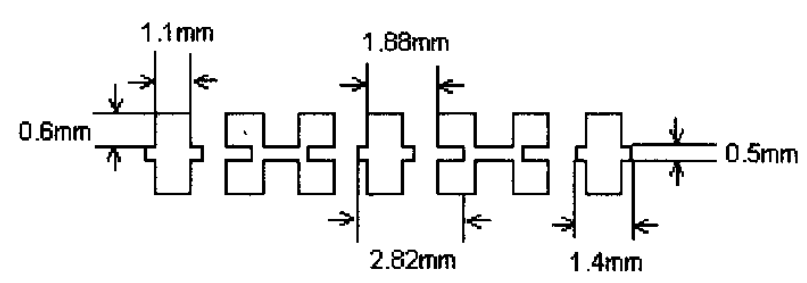

$C$

FIGURE 2 Patterns of the three proposed DGSs. 


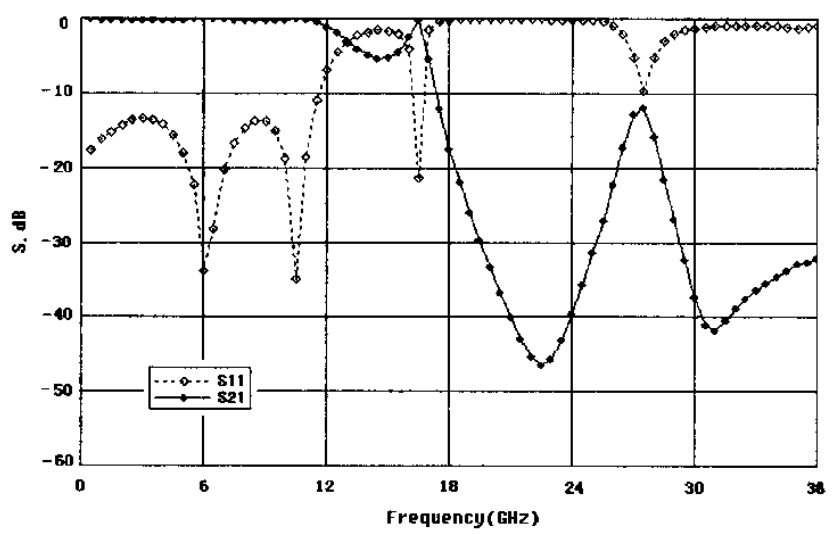

a

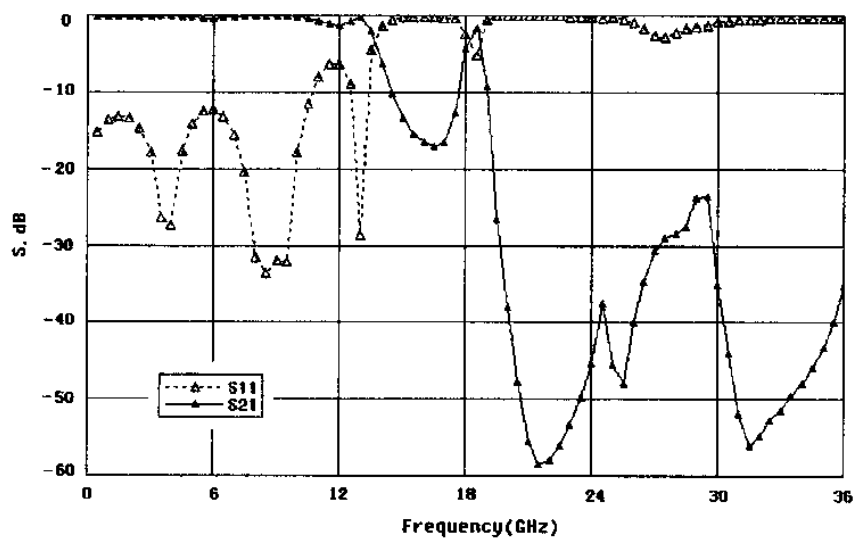

$b$

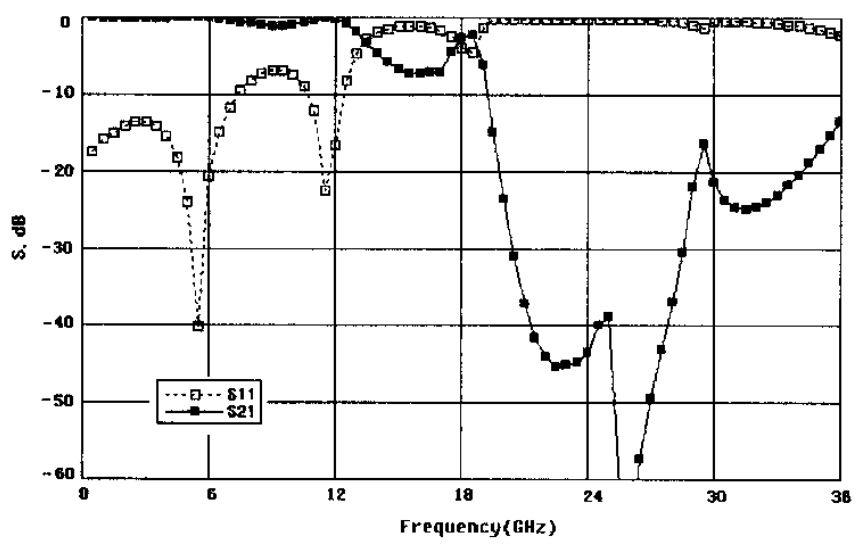

$c$

FIGURE 3 Simulated S parameters of the microstrip line with the three DGSs. 


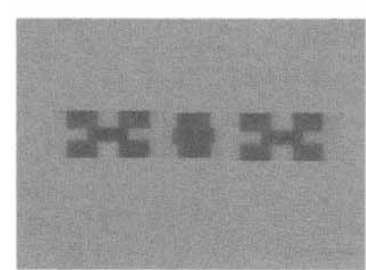

a

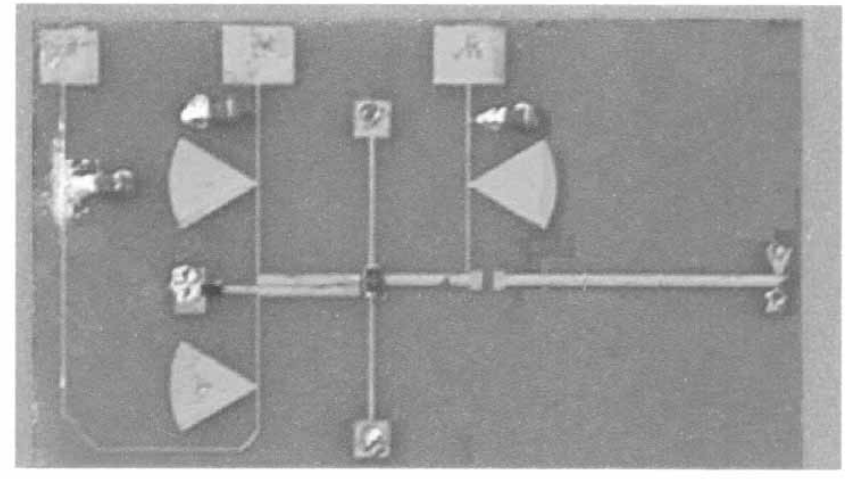

$b$

FIGURE 4 (a) DGS pattern on the ground plane at the output. (b) Photograph of the VCO with DGS.

\section{DESIGN OF THE KU-BAND VCO}

We designed and fabricated two V COs using A Ipha A FM 04P2-000 G aA s M ESFET and A Ipha G M V 982-000 flip-chip varactors for the comparison of output power for two cases: with DGS made up of pattern $b$ shown in Figure $2 b$ and without DGS. The fabricated VCO with the DGS is shown in Figure 4. A common source configuration is used to generate strong negative resistance by inductive feedback [10]. The recommended bias conditions are $V_{G S}=-1 \mathrm{~V}$ and $V_{D S}=5 \mathrm{~V}$ from its data sheets. The operating frequency band of the VCO is from 12.2 to $12.5 \mathrm{GHz}$. The VCO circuit and DGS patterns are realized at the same time by an etching process.

\section{MEASURED RESULTS}

The measured spectrum of VCO with DGS is shown in Figure 5. The output frequency and power are $12.422 \mathrm{GHz}$ and $14.89 \mathrm{dBm}$, respectively, while the bias conditions are

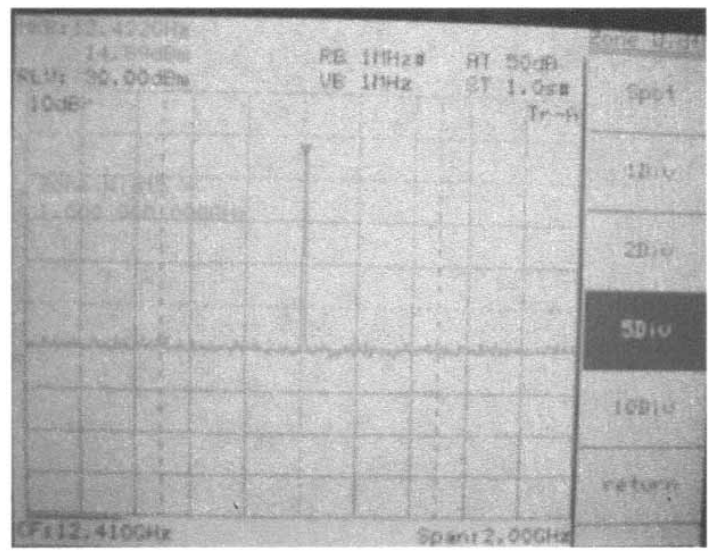

FIGURE 5 Measured spectrum of VCO with DGS. The bias conditions are $V_{\text {varactor }}=-10 \mathrm{~V}, V_{\mathrm{GS}}=-1 \mathrm{~V}$, $\mathrm{V}_{\mathrm{DS}}=5 \mathrm{~V}$, and $\mathrm{I}_{\mathrm{DS}}=45 \mathrm{~mA}$. 


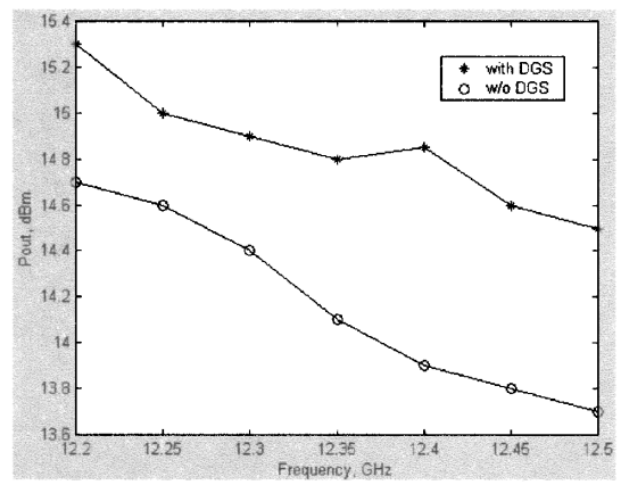

FIGURE 6 M easured output power of VCOs. The bias conditions are $\mathrm{V}_{\text {varactor }}=-10 \mathrm{~V}, \mathrm{~V}_{\mathrm{GS}}=-1 \mathrm{~V}, \mathrm{~V}_{\mathrm{DS}}=5 \mathrm{~V}$, and $I_{D S}=45 \mathrm{~mA}$.

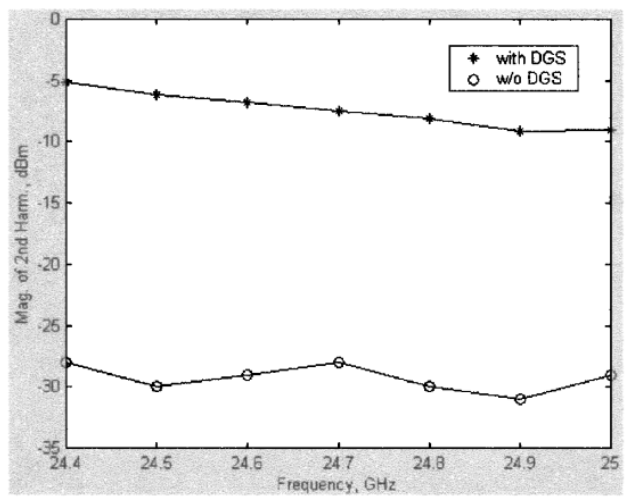

FIGURE 7 M easured 2nd harmonic of VCOs. The bias conditions are $V_{\text {varactor }}=-10 \mathrm{~V}, \mathrm{~V}_{\mathrm{GS}}=-1 \mathrm{~V}, \mathrm{~V}_{\mathrm{DS}}=5 \mathrm{~V}$, and $I_{D S}=45 \mathrm{~mA}$.

$\mathrm{V}_{\text {varactor }}=-10 \mathrm{~V}, \mathrm{~V}_{\mathrm{GS}}=-1 \mathrm{~V}, \mathrm{~V}_{\mathrm{DS}}=5 \mathrm{~V}$, and $\mathrm{I}_{\mathrm{DS}}=45 \mathrm{~mA}$. A comparison of the measured output powers between the VCOs with and without DGS are shown in Figure 6 . The improvement in output power is $0.3-0.8 \mathrm{dBm}$ and is not noticeable because the magnitude of the second harmonic is absolutely small. The output power is improved by $3-5 \%$. A comparison of the measured second harmonic between the VCOs with and without DGS are shown in Figure 7. Figure 7 shows that the second harmonic of the VCO without DGS is -5 to $-9 \mathrm{dBm}$, while it was from -28 to $-31 \mathrm{dBm}$ with DGS. The second harmonic was suppressed more than $-20 \mathrm{dBm}$ at the output and yields improved output power. This means that DGS terminates the second harmonic perfectly. The measurements were performed with a vector network analyzer (HP 8722D).

\section{CONCLUSIONS}

A novel defected ground structure (DGS), which is connected in parallel two periodic structures which have different center frequency of the stopband, is proposed in this paper. V COs with and without the proposed DGS are investigated. From the measured results, the output powers of 
VCO with DGS are improved by $3-5 \%$. The second harmonic of the VCO without DGS waS from -5 to $-9 \mathrm{dBm}$, while it was from -28 to $-31 \mathrm{dBm}$ with DGS. The second harmonic was suppressed more than $-20 \mathrm{~dB}$ m at the output and yields improved output power. That is to say, DGS terminates the second harmonic perfectly. Compared with the conventional PBGs, it is much easier to design and fabricate DGS. Due to its simple fabrication process and planar type, DGS is expected to be applied widely for M IC and M M IC applications.

\section{References}

[1] Radisic, V., Rian, Y. X., Roccioli, R. and Itoh, T. (1998). Novel 2-D photonic bandgap structure for microstrip lines. IEEE Microwave and Guided Wave Letters, 8, 69-71.

[2] Laso, M. G. (2000). Novel wide and photonic bandgap structure. Microwave and Optical Tech. Lett., 24, 357-360.

[3] Caloz, C. and Itoh, T. (2002). Multilayer and anisotropic planar compact PBG structure for microstrip application. IEEE Trans. M TT, 50, 2206-2208.

[4] Kim, T. and Seo, C. (2000). A novel photonic bandgap structure for low-pass filter of wide stopband. IEEE Microwave and Guided Wave Letters, 10, 13-15.

[5] A hn, D., Park, J. S., K im, C. S., K im, J., Qian, Y. X. and Itoh, T. (2001). A design of the low-pass filter using the novel microstrip defected ground structure. IEEE Trans. MTT, 49, 86-93.

[6] Lim, J. S., K im, C. S., L ee, Y. T., A hn, D. and Nam, S. (2002). D esign of lowpass filters using defected ground structure and compensated micostrip line. Electronics Letters, 38, 1357-1358.

[7] Lim, J. S., K im, H. S., A hn, D. and Nam, S. (2001). A power amplifier with efficiency improved using defected ground structure. IEEE Microwave and Guided Wave Letters, 11, 170-172.

[8] Lim, J. S., Park, J. S., Lee, Y. T., A hn, D. and Nam, S. (2002). A pplication of defected ground structure in reducing the size of amplifiers. IEEE Microwave and Guided Wave Letters, 12, 261-263.

[9] Lee, Y. T., Lim, J. S., Park, J. S., A hn, D. and Nam, S. (2002). A novel phase noise reduction technique in oscillators using defected ground structure. IEEE M icrowave and Guided Wave Letters, 12, 39-41.

[10] Soares, R. (1988). GaAs MESFET Circuit Design. A rtech House, B oston and London. 

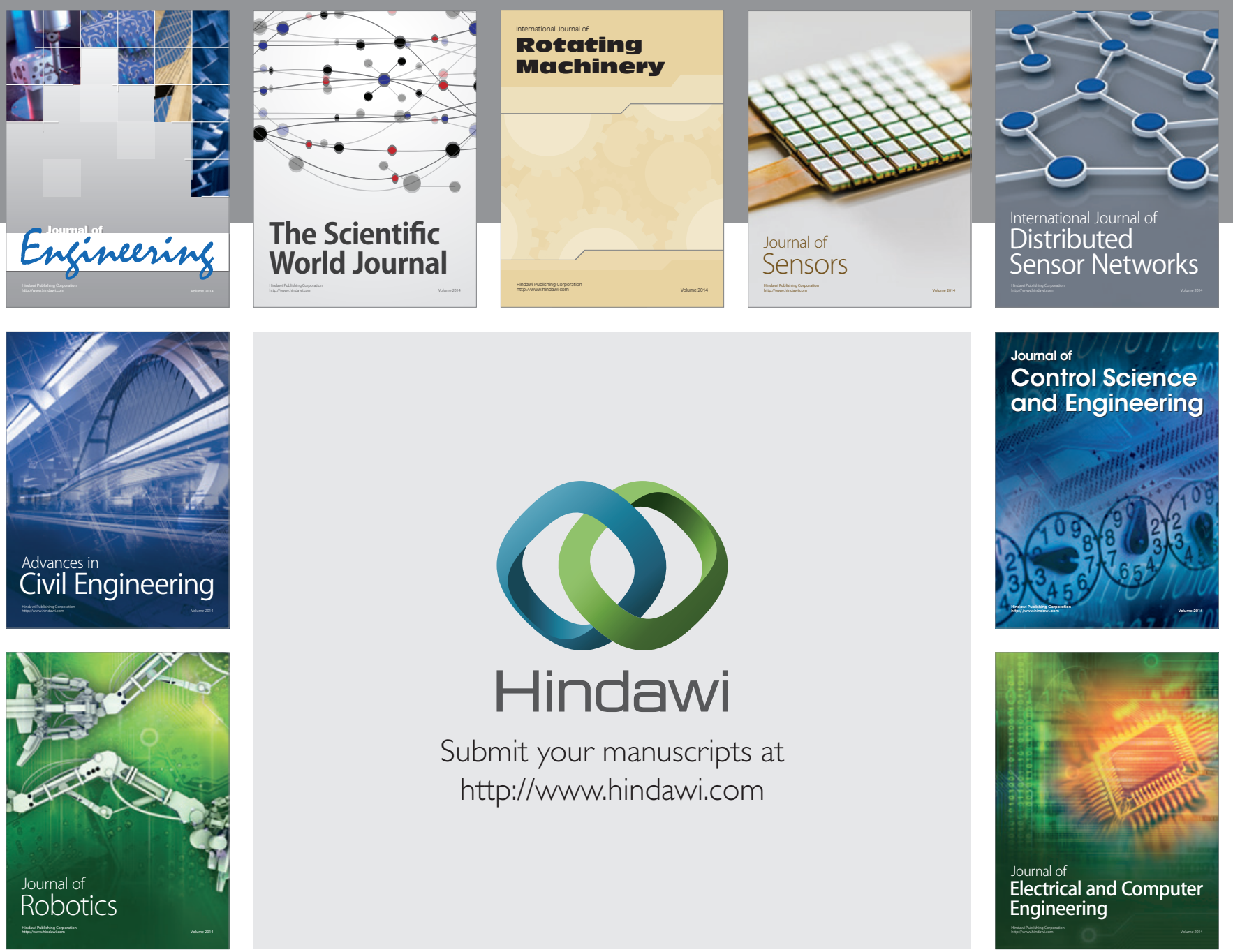

Submit your manuscripts at

http://www.hindawi.com
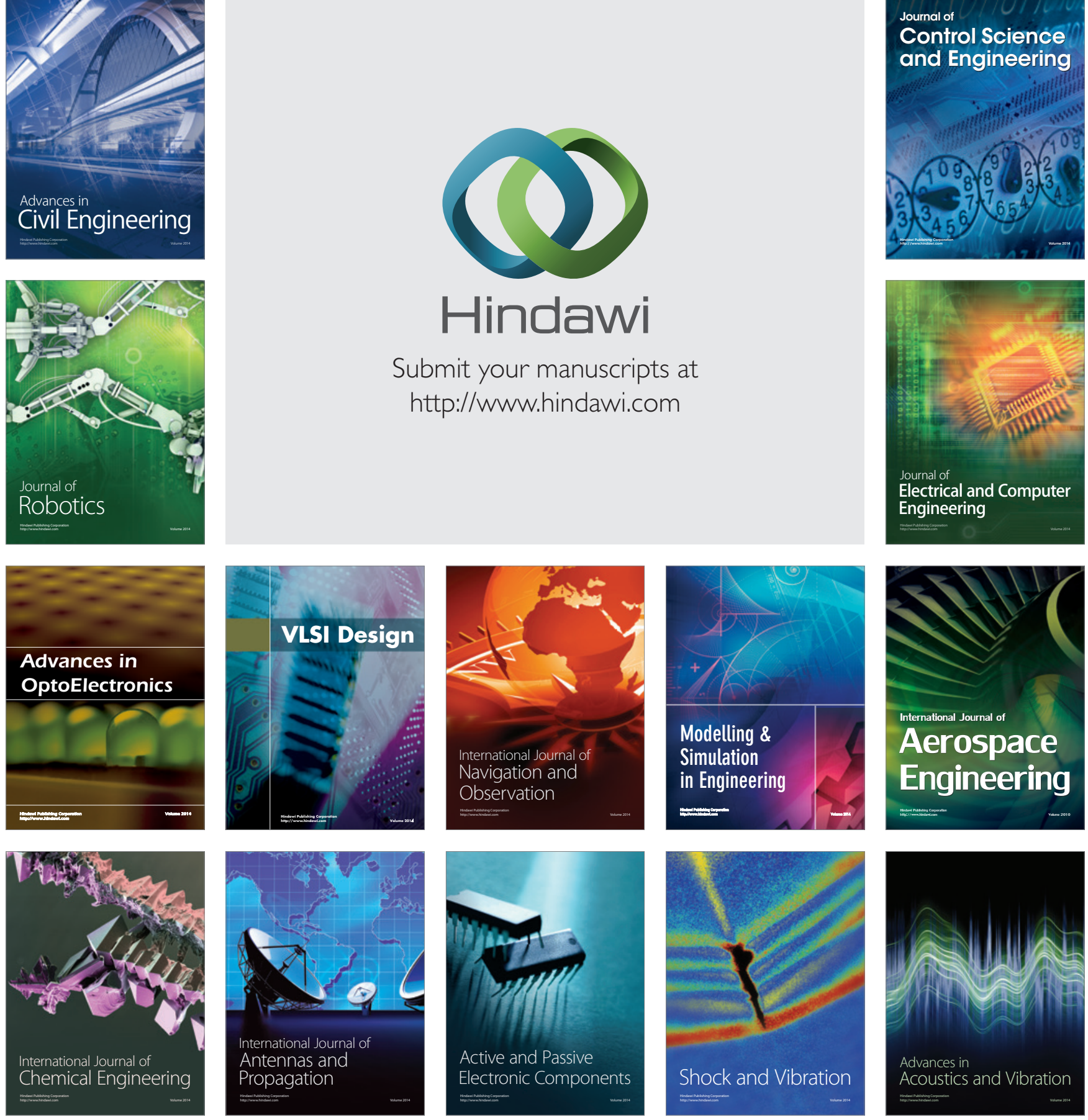